\title{
Maternal vitamin D and $E$ intakes during pregnancy are associated with asthma in children
}

\author{
Keith M. Allan ${ }^{1}$, Nanda Prabhu ${ }^{1}$, Leone C.A. Craig ${ }^{2}$, Geraldine McNeill ${ }^{2}$, \\ Bradley Kirby ${ }^{1}$, James McLay ${ }^{1}$, Peter J. Helms ${ }^{1}$, Jon G. Ayres ${ }^{3}$, \\ Anthony Seaton ${ }^{4}$, Stephen W. Turner ${ }^{1}$ and Graham Devereux ${ }^{1}$
}

Affiliations: ${ }^{1}$ Dept of Child Health, University of Aberdeen, Aberdeen, UK. ${ }^{2}$ Public Health Nutrition Group, University of Aberdeen, Aberdeen, UK. ${ }^{3}$ Institute of Occupational and Environmental Medicine, University of Birmingham, Birmingham, UK. ${ }^{4}$ Dept of Environmental and Occupational Medicine, University of Aberdeen, Aberdeen, UK.

Correspondence: Graham Devereux, Child Health, Royal Aberdeen Children's Hospital, Aberdeen, AB25 2ZG, UK. E-mail: g.devereux@abdn.ac.uk

ABSTRACT Are maternal vitamin D and E intakes during pregnancy associated with asthma in 10-yearold children?

In a longitudinal study of 1924 children born to women recruited during pregnancy, maternal vitamin D intake during pregnancy was assessed by the Food Frequency Questionnaire (FFQ) and vitamin E by FFQ and plasma $\alpha$-tocopherol; respiratory questionnaires were completed for the 10 -year-old children. Their treatment for asthma was also ascertained using administrative data. Longitudinal analyses included data collected at $1,2,5$ and 10 years.

Symptom data were available for 934 (49\%) children and use of asthma medication for 1748 (91\%). In the children maternal vitamin D intake during pregnancy was negatively associated with doctor-diagnosed asthma at 10 years of age (OR per intake quintile $0.86,95 \%$ CI $0.74-0.99$ ) and over the first 10 years (hazard ratio $0.90,95 \%$ CI $0.81-1.00$ ). Maternal plasma $\alpha$-tocopherol at 11 weeks gestation was negatively associated with children receiving asthma treatment (OR per standard deviation increase 0.52, 95\% CI 0.31-0.87). Maternal vitamin E intake was negatively associated with doctor-diagnosed asthma (OR 0.89 , 95\% CI 0.81-0.99) in the first 10 years.

Low maternal vitamin $\mathrm{D}$ and $\mathrm{E}$ intakes during pregnancy are associated with increased risk of children developing asthma in the first 10 years of life. These associations may have significant public health implications.

@ERSpublications

Children born to women with low vitamin $\mathrm{D}$ and $\mathrm{E}$ intakes during pregnancy are more likely to develop asthma by age $10 \mathrm{http}: / /$ ow.ly/CmDo8

This article has supplementary material available from erj.ersjournals.com

Received: June 042014 | Accepted after revision: Sept 252014 | First published online: Oct 302014

Support statement: The 10-year follow-up of this cohort was funded by the UK Medical Research Council (grant number: 80219), the recruitment and follow-up at 1,2 and 5 years was funded by Asthma UK (grant numbers: 00/011 and 02/017).

Conflict of interest: Disclosures can be found alongside the online version of this article at erj.ersjournals.com

Copyright CERS 2015 


\section{Introduction}

It has been hypothesised that changing diet contributed to the increase in asthma and atopic disease observed in economically developed countries in the latter decades of the 20th century [1]. Recognising that early life factors play critical roles in the development of asthma and atopic disease, studies have related maternal dietary status during pregnancy to the development of childhood asthma and atopic disease. Several have reported lower maternal dietary vitamin D intake during pregnancy to be associated with an increased risk of wheeze, asthma, eczema and atopic sensitisation in children up to the age of 7 years [2-6]; although maternal and/or umbilical cord blood 25-hydroxy-vitamin $\mathrm{D}_{3}\left(25(\mathrm{OH}) \mathrm{D}_{3}\right)$ has been reported to be either not [7-10], adversely [11-13] or beneficially [14-17] associated with respiratory and/ or atopic outcomes up to the age of 9 years. Several studies have reported lower maternal dietary vitamin E intake during pregnancy to be either associated with an increased risk of wheezing at age 2 years $[18,19]$ or not associated with asthma or atopic outcomes at age 1,5 or 7 years [20-22].

We have established a cohort of children born to women recruited during pregnancy, to test the hypothesis that maternal nutrient intake during pregnancy influences susceptibility to childhood asthma. Previously we reported that children whose mothers' vitamin D intake during pregnancy had been relatively low were more likely to wheeze at 5 years of age [23]. We have also reported that children born to women with a low vitamin $\mathrm{E}$ intake during pregnancy were more likely to wheeze at 2 and 5 years of age, and more likely to have asthma at 5 years of age [24, 25].

We present here the results of the 10-year cohort follow-up, performed to test the hypothesis that the associations between respiratory symptoms at age 5 years with maternal vitamin $\mathrm{D}$ and $\mathrm{E}$ intakes during pregnancy persist into later childhood. In addition to using the conventional methodology employed in previous phases that required active participation, we augmented the conventional follow-up with routinely collected healthcare data.

\section{Methods}

2000 healthy unselected pregnant women attending an antenatal clinic, at median 11 weeks gestation, were recruited between 1997 and 1999 [24, 25]. At enrolment, an interviewer administered a questionnaire, skin prick testing (SPT) was performed and a non-fasting blood sample obtained. At 32 weeks gestation, dietary intake over the preceding 3 months was assessed using version 5.4 of the Scottish Collaborative Group Food Frequency Questionnaire (FFQ) [26]. In women of childbearing age the correlation coefficients between intakes of vitamin $\mathrm{D}$ and $\mathrm{E}$ derived by this questionnaire and 4-day weighed records were $0.38(\mathrm{p}<0.001)$ and $0.52(\mathrm{p}<0.001)$, respectively [27].

Plasma $\alpha$-tocopherol was determined by normal phase high-performance liquid chromatography.

\section{Assessment of children}

In this study singletons born to the recruited women were followed up at 10 years. In the month of the child's 10th birthday, an ISAAC (International Study of Asthma and Allergies in Childhood) based questionnaire $[24,25]$ was mailed to all participating families, with a single reminder if necessary. Wheeze was defined by an affirmative response to the question "has your child had wheezing or whistling in the chest in the last 12 months?" Similar questions enquired about whether the child "ever wheezed" and "wheezed in the absence of a cold". Doctor-diagnosed asthma was defined by positive responses to the questions "has your child ever suffered from asthma?" and "was this confirmed by a doctor?" Use of asthma medication in the past year was also ascertained. Similar questions enquired about eczema and hay fever. Current asthma was defined as asthma and wheeze in the previous year.

Parents responding to the questionnaire were invited to complete an FFQ assessing the study child's dietary intake and to bring the child for an assessment that included spirometry, SPT and measurement of exhaled nitric oxide $(\mathrm{FeNO})$. These are described in the online supplementary material.

The study was approved by the North of Scotland Research Ethics Committee (08/S0802/19), written parental consent was obtained, and children gave written assent.

\section{Augmented follow-up}

The Prescribing Information System (PIS) database held by the Information Services Division of National Health Service Scotland was used to increase case ascertainment for the outcome "use of medication to treat asthma in the last year". PIS records every item dispensed by a Scottish pharmacy at patient level identifiable by the Community Health Index (CHI) number. For study children resident in Scotland their $\mathrm{CHI}$ number was used to identify if study children were dispensed/not dispensed asthma medication in their 10th year; these data were then linked to the main study database using CHI. 


\section{Statistical analysis}

Asthma, wheeze, eczema and hay fever in the 10-year-old children were related to maternal plasma $\alpha$-tocopherol, and vitamin D and E intakes. For all women completing the FFQ, dietary and supplement intakes were summated, energy-adjusted [28] and divided into fifths. Plasma $\alpha$-tocopherol was logarithmically transformed, adjusted for cholesterol and gestational age, and derived odds ratios presented per standard deviation increase. Unadjusted and adjusted modelling was performed. Adjusted models included potentially confounding covariates selected on: conceptual evidence, for example maternal smoking during pregnancy and maternal atopy ( $\geqslant 1$ positive SPT with a $3 \mathrm{~mm}$ cut-off); birth order; child's sex; maternal age at recruitment and maternal socioeconomic status (SES) at recruitment (using the Scottish Index of Multiple Deprivation [29]); and statistical tests (birth weight, length and head circumference), achieving $\mathrm{p}<0.25$ in univariate analyses [30]. Associations with vitamin $\mathrm{D}$ were adjusted for vitamin $\mathrm{E}$ and vice versa. Further analyses included children's nutrient intake at 10 years of age and replaced area-based SES parameters with alternative metrics based on parental occupation and maternal education. Maternal nutrient intake and status was initially related to 10 -year outcomes cross-sectionally using logistic regression. The 10-year data were then combined with data collected at $1(\mathrm{n}=1511), 2$ $(n=1373)$ and 5 years $(n=1253)$. Generalised estimating equations with exchangeable correlation structures were used to relate maternal nutrient intake and status and repeated measurements of childhood wheeze. Discrete-time hazards modelling was used to analyse new incident asthma, eczema and hay fever. Analyses were performed using IBM SPSS Statistics for Windows, v21.0 (IBM Corp., Armonk, NY, USA).

\section{Results}

Figure 1 details the number of mothers and children who actively participated at 10 years and those included in the augmented follow-up. Of the 2000 pregnant women recruited, 1924 delivered a live singleton, but because of withdrawals 1878 were sent questionnaires. Plasma $\alpha$-tocopherol was quantified in 1917 (99.6\%) women at 11 weeks gestation and 1717 (89.2\%) women provided FFQ data at 32 weeks gestation.

Questionnaires were returned for 934 (49\%) of the 1924 10-year-old children. Maternal dietary and $\alpha$-tocopherol data during pregnancy were available for 909 (97\%) and 932 (99\%) children, respectively. The children's FFQ was completed by 595 children/parents and 449 attended a clinical assessment; 433 performed spirometry, 432 underwent SPT, 419 had their FeNO quantified and 380 underwent methacholine challenge.

Tables 1 and E1 demonstrate that the mothers of children actively participating in the questionnaire and clinical phases were less likely to smoke, were older, of higher SES, less likely to have wheezed, and had higher plasma ascorbate and $\beta$-carotene, whereas participating children were more likely to be girls and of higher birth weight. Active participation was not associated with maternal vitamin D or E intakes, maternal asthma or atopy, or plasma $\alpha$-tocopherol.

The PIS database revealed that 1660 study children resided in Scotland. Of the remaining 218 non-resident children 88 returned postal questionnaires. The augmented follow-up comprised 1748 (91\%) children out of the original 1924, comprising 1660 with PIS data and the 88 resident outside Scotland with questionnaire data.

The prevalence of wheezing, asthma, eczema, and hay fever in the 10-year-old study children are outlined in table 2. Use of asthma medication in the past year was reported by 109 (11.7\%) of postal questionnaire respondents, whereas PIS revealed that $161(9.2 \%)$ of study children were dispensed asthma medication in the year before their 10th birthday. Paired PIS and questionnaire data were available for 839 children, 78 children were treated for asthma in both the questionnaire and PIS, whereas 731 were not treated for asthma in both PIS and questionnaire. The PIS database with respect to the questionnaire had sensitivity $87 \%$, specificity $99 \%$, positive predictive value $90 \%$ and negative predictive value $98 \%$, and a Kappa of 0.87 .

\section{Maternal vitamin D intake}

In cross-sectional analyses of the 934 children with questionnaire data, low maternal vitamin D intake during pregnancy was associated with an increased likelihood of wheezing and doctor-diagnosed asthma in 10-year-old children (table 3). Analysis of the augmented follow-up indicated that decreasing maternal vitamin D intake was associated with increased likelihood of treated asthma, but this was not significant ( ptrend=0.092). Longitudinal analysis of the combined 1, 2, 5 and 10 year data demonstrated that low maternal vitamin D intake during pregnancy was associated with an increased likelihood of wheeze, doctor-diagnosed asthma and asthma with wheeze in children during their first 10 years of life. Maternal vitamin D intake was not associated with childhood eczema or hay fever outcomes (table E2). 


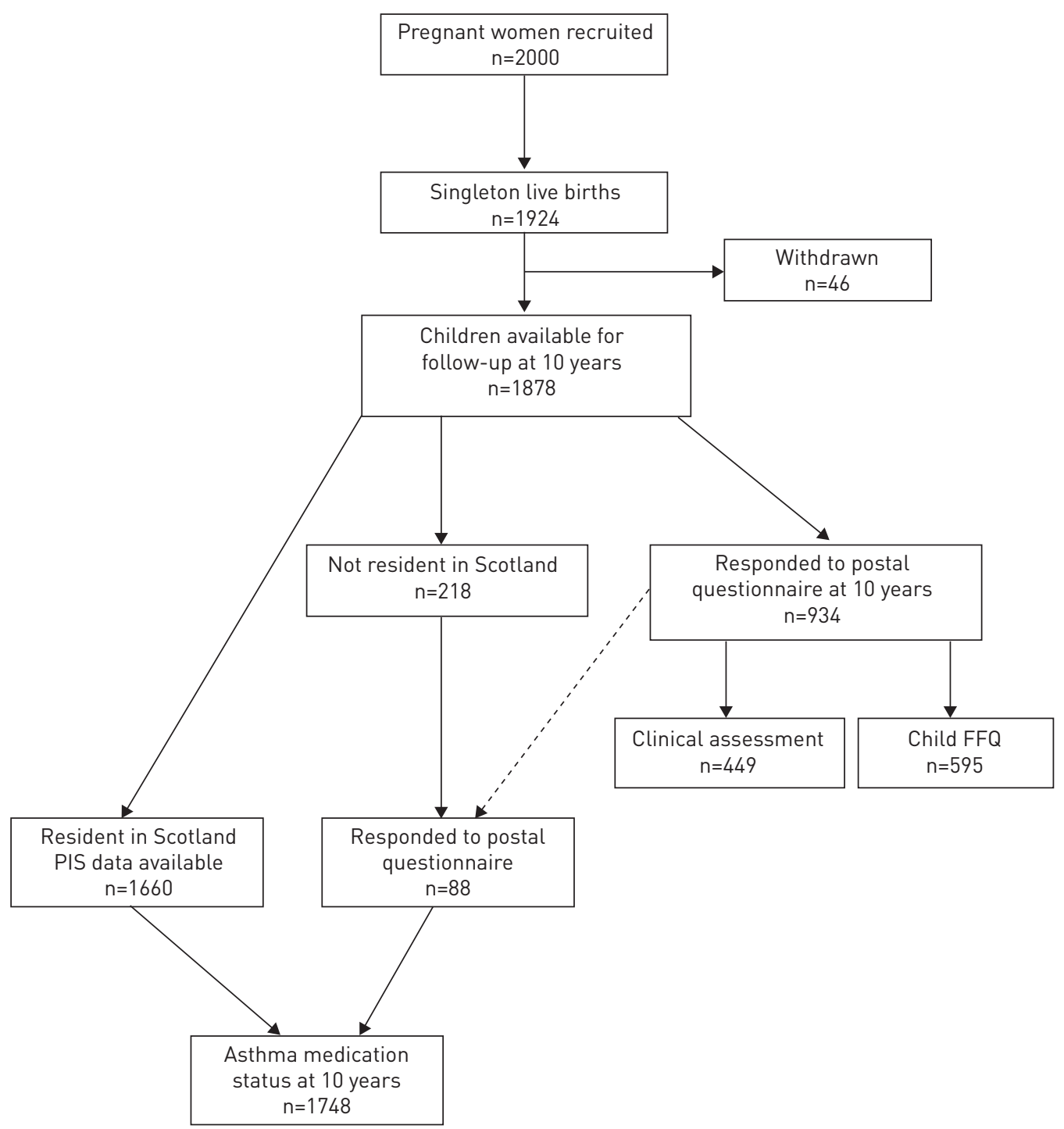

FIGURE 1 Summary of children involved in the various aspects of the 10-year follow-up. FFQ: Food Frequency Questionnaire; PIS: Prescribing Information System.

\section{Maternal vitamin E status}

In cross-sectional analyses of the 934 children with questionnaire data, maternal vitamin $\mathrm{E}$ intake during pregnancy was not associated with wheeze and asthma in 10-year-old children; there was a nonsignificant ( $p=0.096$ ) association between low maternal plasma $\alpha$-tocopherol and increased use of asthma medication at 10 years of age (tables 4 and 5). Analysis of the augmented follow-up indicated that decreasing maternal plasma $\alpha$-tocopherol was associated with an increased likelihood of 10-year-old children being dispensed asthma medication. Longitudinal analysis of the 1,2,5 and 10 year data demonstrated that low maternal vitamin $\mathrm{E}$ intake was associated with an increased likelihood of wheeze, doctor-diagnosed asthma and asthma with wheeze in children during their first 10 years of life. Maternal vitamin E status was not associated with childhood eczema or hay fever outcomes (table E3).

\section{Further analyses}

Energy adjusted maternal vitamin D and E intakes were weakly correlated (Pearson's correlation coefficient $0.29, \mathrm{p}<0.001)$. There was no statistically significant interaction between maternal vitamin $\mathrm{D}$ and $\mathrm{E}$ intakes and childhood outcomes. Dichotomising maternal vitamin D and E intakes about the median value into "high" and "low" intakes demonstrated an additive effect, with children born to women with vitamin $\mathrm{D}^{\text {low } / ~}$ $\mathrm{E}^{\text {low }}$ intakes being more likely to wheeze in the first 10 years (OR 1.48, 95\% CI 1.11-1.95) than children born to women with vitamin $\mathrm{D}^{\text {high }} / \mathrm{E}^{\text {high }}$, vitamin $\mathrm{D}^{\text {high }} / \mathrm{E}^{\text {low }}$ or vitamin $\mathrm{D}^{\text {low }} / \mathrm{E}^{\text {high }}$ intakes. Similarly children born to women with vitamin $\mathrm{D}^{\text {low }} / \mathrm{E}^{\text {low }}$ intakes were more likely to be diagnosed with asthma in the first 
TABLE 1 Characteristics of mothers at recruitment, those responding to the 10-year follow-up questionnaire and those for whom routinely collected healthcare data were available for their child at 10 years of age

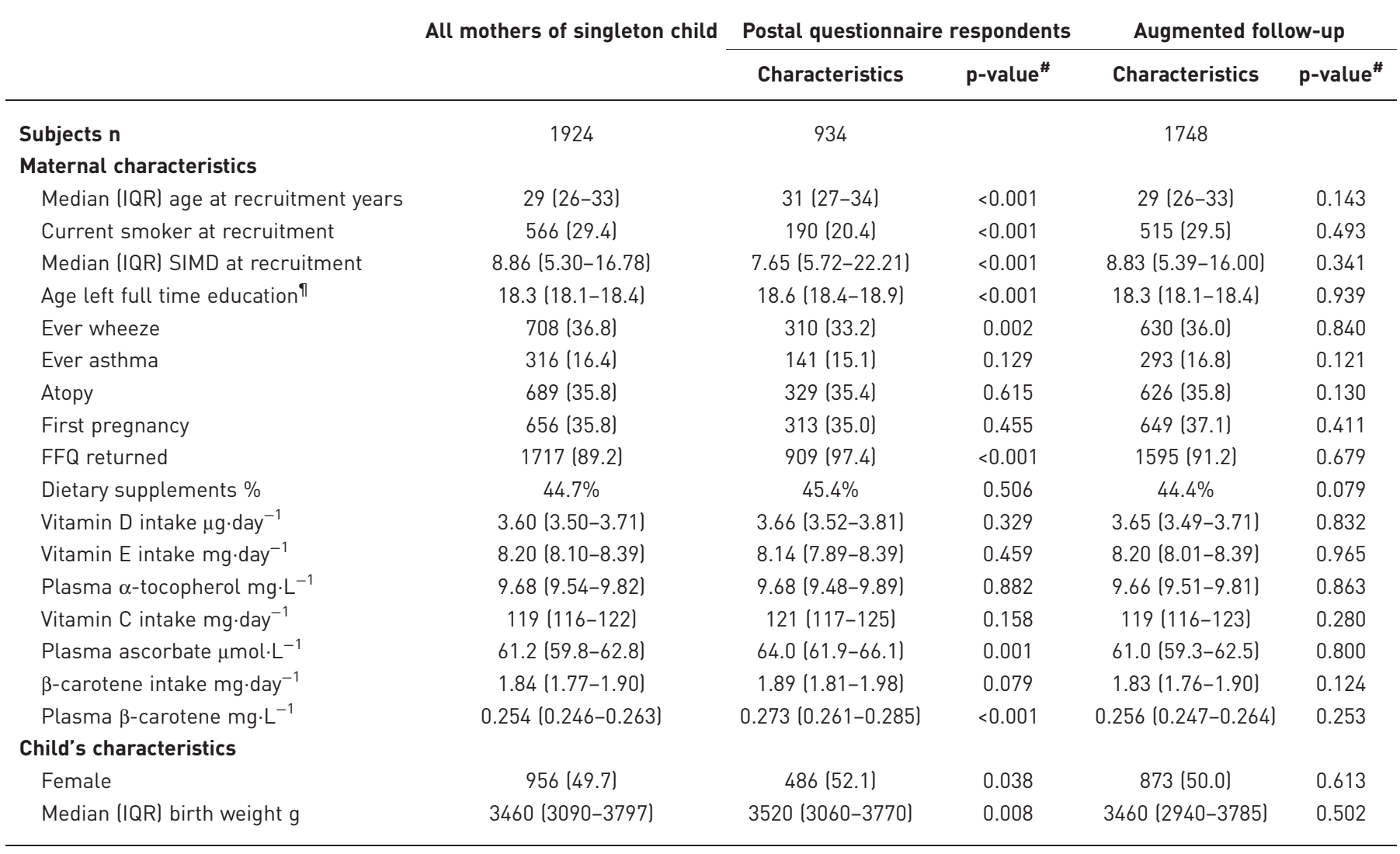

Data are presented as $\mathrm{n}(\%)$ or geometric mean $(95 \% \mathrm{CI}$ ), unless otherwise stated. IQR: interquartile range; SIMD: Scottish Index of Multiple Deprivation; FFQ: Food Frequency Questionnaire. " : p-values for those with data versus remaining cohort members, using Chi-squared, MannWhitney or t-tests; ${ }^{\text {१: }}$ data for age left full time education are presented as mean $(95 \% \mathrm{CI})$.

TABLE 2 Prevalence of wheeze, asthma, eczema and hay fever in 10 year-old children followed up by postal questionnaire and by routinely collected healthcare data

Postal questionnaire

Augmented follow-up

\begin{tabular}{|c|c|c|}
\hline Subjects n & 934 & 1748 \\
\hline Ever wheezed & $205(22.1)$ & \\
\hline Wheezed in past 12 months & $110(11.9)$ & \\
\hline Wheezed in the absence of a cold in past 12 months & $75(8.1)$ & \\
\hline Ever had asthma & $142(15.2)$ & \\
\hline Doctor confirmed asthma & $136(14.8)$ & \\
\hline Medication to treat asthma in past 12 months & $109(11.7)$ & $161(9.2)$ \\
\hline Asthma and wheeze in previous 12 months & 78 (8.5) & \\
\hline Ever eczema & $298(32.6)$ & \\
\hline Doctor confirmed eczema & $244(32.0)$ & \\
\hline Medication to treat eczema in past 12 months & $81(8.7)$ & \\
\hline Ever hay fever & $253(27.2)$ & \\
\hline Doctor confirmed hay fever & $119(13.1)$ & \\
\hline Medication to treat hay fever in past 12 months & $212(23.0)$ & \\
\hline
\end{tabular}

Data are presented as $\mathrm{n}(\%)$, unless otherwise stated. 
TABLE 3 Associations between total maternal vitamin D intake during pregnancy and wheezing and asthma outcomes in 10-year-old children

Quintiles of energy adjusted maternal vitamin D intake

ptrend

\begin{tabular}{|c|c|c|c|c|c|c|}
\hline Quintile & Q1 & Q2 & Q3 & Q4 & Q5 & \\
\hline Intake $\mu \mathrm{g} \cdot$ day $^{-1}$ & & & & & & \\
\hline \multicolumn{7}{|l|}{ Postal questionnaire } \\
\hline Ever wheezed & 1 & $0.98(0.60-1.61)$ & $0.74(0.44-1.23)$ & $0.58(0.34-0.99)$ & $0.51(0.30-0.87)$ & 0.002 \\
\hline Wheeze in past year & 1 & $1.76(0.94-3.30)$ & $1.12(0.58-2.18)$ & $0.68(0.33-1.41)$ & $0.59(0.28-1.24)$ & 0.009 \\
\hline Wheezed in absence of a cold in past year & 1 & $1.27(0.62-2.60)$ & $0.58(0.25-1.33)$ & $0.60(0.26-1.35)$ & $0.63(0.28-1.41)$ & 0.056 \\
\hline Asthma and wheeze in past year & 1 & $1.33(0.65-2.71)$ & $0.74(0.34-1.61)$ & $0.64(0.28-1.44)$ & $0.63(0.28-1.44)$ & 0.092 \\
\hline Medication to treat asthma in past year & 1 & $1.08(0.57-2.04)$ & $0.76(0.39-1.48)$ & $0.67(0.34-1.34)$ & $0.74(0.37-1.46)$ & 0.158 \\
\hline \multicolumn{7}{|l|}{ Augmented follow-upף } \\
\hline Dispensed asthma medication in past year & 1 & $0.97(0.58-1.64)$ & $0.83(0.48-1.42)$ & $0.55(0.30-1.00)$ & $0.76(0.43-1.34)$ & 0.092 \\
\hline \multicolumn{7}{|l|}{ Longitudinal $^{+}$} \\
\hline
\end{tabular}

Data are present as adjusted odds ratio (95\% Cl), unless otherwise stated. Odds ratios were adjusted for maternal smoking during pregnancy, maternal atopy, birth order, child's sex, maternal age at recruitment, Scottish Index of Multiple Deprivation, birth weight, birth crown-heel length, birth head circumference and maternal vitamin $E$ intake. ${ }^{\#}: n=934 ;{ }^{\uparrow}: n=1748 ;{ }^{+}: 1,2,5$ and 10 year data; ${ }^{\S}$ : generalised estimating equations; ${ }^{f}$ : discrete hazards modelling, hazard ratio.

TABLE 4 Associations between total maternal vitamin E intake during pregnancy and wheezing and asthma outcomes in 10-year-old children

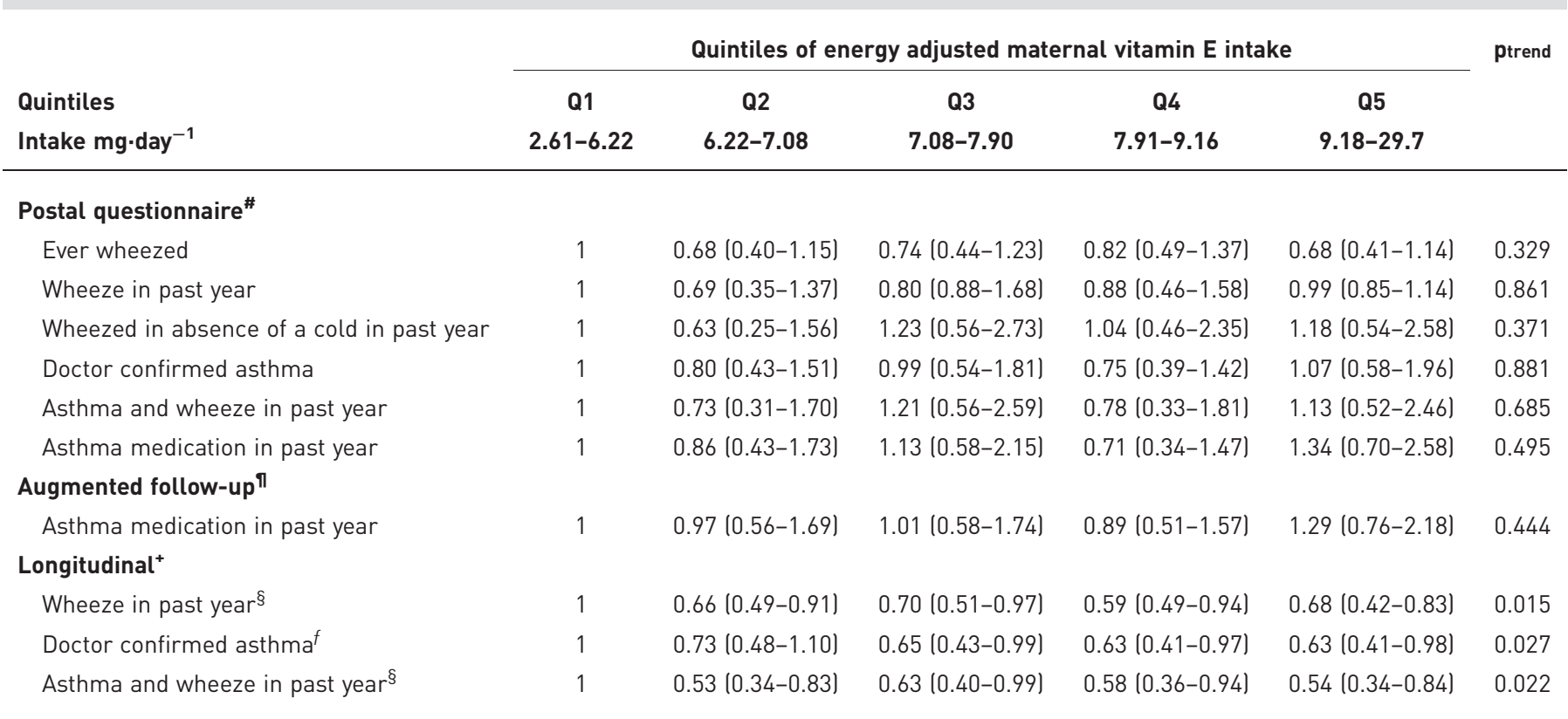

Data are presented as adjusted odds ratio $(95 \% \mathrm{Cl})$, unless otherwise stated. Odds ratios were adjusted for maternal smoking during pregnancy, maternal atopy, birth order, child's sex, maternal age at recruitment, Scottish Index of Multiple Deprivation, birth weight, birth crown-heel length, birth head circumference and maternal vitamin D intake. ${ }^{\#}: n=934 ;{ }^{\uparrow}: n=1748 ;{ }^{+}: 1,2,5$ and 10 year data; ${ }^{\S}$ : generalised estimating equations; ${ }^{f}$ : discrete hazards modelling, hazard ratio. 
TABLE 5 Associations between plasma $\alpha$-tocopherol during pregnancy and wheezing and asthma outcomes in 10-year-old children

\begin{tabular}{|c|c|c|}
\hline & \multicolumn{2}{|c|}{ Maternal $\alpha$-tocopherol $\mu \mathrm{g} \cdot \mathrm{mL}^{-1}$} \\
\hline & Adjusted OR $(95 \% \mathrm{CI})^{\#, \pi}$ & p-value \\
\hline \multicolumn{3}{|l|}{ Postal questionnaire ${ }^{+}$} \\
\hline Ever wheezed & $0.71(0.43-1.19)$ & 0.198 \\
\hline Wheeze in past year & $0.74(0.39-1.41)$ & 0.365 \\
\hline Wheezed in absence of a cold in past year & $0.76(0.35-1.64)$ & 0.477 \\
\hline Doctor confirmed asthma & $0.64(0.35-1.17)$ & 0.148 \\
\hline Asthma and wheeze in past year & $0.80(0.37-1.71)$ & 0.563 \\
\hline Asthma medication in past year & $0.58(0.31-1.10)$ & 0.096 \\
\hline \multicolumn{3}{|l|}{ Augmented follow-up ${ }^{\S}$} \\
\hline Asthma medication in past year & $0.52(0.31-0.87)$ & 0.013 \\
\hline \multicolumn{3}{|l|}{ Longitudinal ${ }^{f}$} \\
\hline Wheeze in past year ${ }^{\# \#}$ & $0.80(0.57-1.12)$ & 0.194 \\
\hline Doctor confirmed asthma & $0.79(0.51-1.22)$ & 0.284 \\
\hline Asthma and wheeze in past year $\# \#$ & $0.76(0.49-1.18)$ & 0.219 \\
\hline \multicolumn{3}{|c|}{$\begin{array}{l}\# \text { : adjusted for maternal smoking during pregnancy, maternal atopy, birth order, child's sex, maternal age } \\
\text { at recruitment, Scottish Index of Multiple Deprivation, birth weight, birth crown-heel length, birth head } \\
\text { circumference and maternal vitamin } D \text { intake; }{ }^{\uparrow} \text { : OR expressed per standard deviation increase in } \\
\alpha \text {-tocopherol, adjusted for plasma cholesterol and gestational age when sample taken; }{ }^{+}: n=934 ;{ }^{\S}: n=1748 ; \\
f: 1,2,5 \text { and } 10 \text { year data; }{ }^{\# \#} \text { : generalised estimating equations; }{ }^{\text {ๆा }} \text { : discrete hazards modelling, hazard } \\
\text { ratio. }\end{array}$} \\
\hline
\end{tabular}

10 years (OR 1.71, 95\% CI 1.19-2.46) than children born to women with vitamin $\mathrm{D}^{\text {high }} / \mathrm{E}^{\text {high }}$ intakes, or with vitamin $\mathrm{D}^{\text {high }} / \mathrm{E}^{\text {low }}(\mathrm{OR} 1.43,95 \%$ CI $0.97-2.11)$ or vitamin $\mathrm{D}^{\text {low }} / \mathrm{E}^{\text {high }}(\mathrm{OR} 1.55,95 \%$ CI $1.07-2.25$ ) intakes (unadjusted data presented as figure E1). Maternal vitamin D or E intakes during pregnancy were not associated with lung function, methacholine responsiveness, FeNO or atopic status in the 449 children attending clinical assessment (results not presented). Use of non-area based SES metrics or presence of household smokers when the child was 10 years-old did not significantly alter any of the reported associations. Dietary FFQ data for 595 10-year-old children were available; the vitamin D and E intakes of these children were not associated with wheeze, asthma, eczema or hay fever outcomes (tables E4 and E5).

\section{Discussion}

We believe this is the longest follow-up of a birth cohort relating maternal vitamin D and E intake/status during pregnancy to childhood respiratory outcomes. This cohort reported low maternal vitamin D intake to be associated with increased risk of childhood wheezing at 5 years of age and low maternal vitamin $\mathrm{E}$ intake to be associated with an increased likelihood of childhood wheezing and asthma at 5 years of age $[23,25]$. We now show that 10 -year-old children are more likely to wheeze and be diagnosed with asthma if their mother had a low vitamin D intake. In addition, children are more likely to wheeze and have diagnosed asthma by 10 years-old if their mother had a low vitamin E intake. 10-year-old children are also more likely to be treated for asthma if their mother had low first trimester plasma $\alpha$-tocopherol. The vitamin $\mathrm{D}$ and $\mathrm{E}$ associations were independent of each other and their effects appeared to be additive.

The beneficial associations with maternal vitamin D intake during pregnancy reported here extend previous observations made up to 7 years of age. Vitamin D has modulatory effects on innate and adaptive immunity, particularly regulatory T-cells [31], and higher maternal vitamin $\mathrm{D}$ intake during pregnancy has been associated with reduced likelihood of wheeze and eczema at 2 years of age [5], recurrent wheeze at 3 years of age [2], asthma and food allergen sensitisation at 5 years of age [3], and asthma at 7 years of age [6]. While maternal vitamin $\mathrm{D}$ intake has been consistently beneficially associated with childhood wheeze/asthma, studies of maternal and/or umbilical plasma $25(\mathrm{OH}) \mathrm{D}_{3}$ have been inconsistent [7-17]. Dietary intake typically contributes about $10 \%$ to UK vitamin D status and while studies of plasma $25(\mathrm{OH}) \mathrm{D}_{3}$ truly reflect associations with vitamin $\mathrm{D}$ status, perhaps studies of vitamin $\mathrm{D}$ intake should be interpreted more broadly. Vitamin D is never naturally consumed in isolation, but as part of foods containing many nutrients; indeed it may be the combination of naturally associated nutrients that is important and should perhaps be replicated in intervention studies. Trials of vitamin D supplements during pregnancy are underway; given the inconsistencies between dietary and plasma vitamin $\mathrm{D}$ these may not provide clear answers. A small trial has reported that vitamin $\mathrm{D}$ supplements 
during pregnancy did not reduce wheeze or asthma in 3-year-old children [32]. This trial, however, supplemented from 27 weeks gestation, whereas in the current study maternal habitual vitamin D intake for the 3 months up to 32 weeks gestation was assessed, suggesting perhaps that first and second trimester intervention before 27 weeks gestation may be more appropriate.

The associations we report between maternal vitamin E status during pregnancy and childhood wheeze and asthma are consistent with our findings at 2 and 5 years of age $[24,25]$ and with other studies [18, 19]. Three studies have reported maternal vitamin $\mathrm{E}$ intake not to be associated with childhood outcomes, although one highlighted their poor FFQ validity for vitamin E [21], another's FFQ was not validated for vitamin E [22] and the third only reported 1 year outcomes [20].

Although the associations between maternal vitamin E and childhood wheeze/asthma we report here are consistent, there are some potentially informative discrepancies. Cross-sectional analyses of the questionnaire data revealed no associations with FFQ-derived maternal vitamin $\mathrm{E}$ intake whereas in the cross-sectional augmented follow-up, low maternal plasma $\alpha$-tocopherol was associated with children receiving treatment for asthma. Several factors might have contributed to the discordance between maternal plasma $\alpha$-tocopherol and FFQ-derived vitamin E intake. Plasma $\alpha$-tocopherol and augmented follow-up data were independent of parental recall and available for $91 \%$ of the original cohort, whereas postal questionnaire data were available for $49 \%$ and dependent on recall. A further possible contributory factor is that dietary vitamin E comprises the natural isoforms $\alpha$ - and $\gamma$-tocopherol. In murine eosinophilic allergic lung inflammation $\alpha$-tocopherol is anti-inflammatory, whereas $\gamma$-tocopherol is pro-inflammatory [33]. Perhaps most relevant to the disparity between plasma $\alpha$-tocopherol and vitamin $\mathrm{E}$ intake is that the susceptibility of developing airways to suboptimal vitamin $\mathrm{E}$ may change in utero. Maternal plasma $\alpha$-tocopherol was measured at 11 weeks gestation at the pseudoglandular stage of lung development (7-17 weeks) [34], the period of fastest division of intrasegmental airways, by the end of which the formation of conducting airways and terminal bronchioles is complete. In rat models of fetal hypoplastic lung growth induced in the pseudoglandular phase, maternal vitamin E supplementation accelerates lung growth increasing lung complexity, surface area and bud count [35]. In our study maternal vitamin $\mathrm{E}$ intake was quantified by FFQ at 32 weeks gestation, during the canalicular stage of respiratory bronchiole and alveolar development. It may be that the associations between maternal vitamin $\mathrm{E}$ intake and 5-year wheeze/asthma outcomes were a consequence of effects on pseudoglandular and canalicular developmental stages and that as the children's airways grow, the airway effects of suboptimal vitamin E status on the less critical canalicular phase wane, whereas effects on the critical pseudoglandular phase of airway development remain as evidenced by the association between $\alpha$-tocopherol and the severest forms of wheezing illness, recognised as treated asthma.

The 10-year follow-up had strengths and limitations. Strengths included the use of routinely collected healthcare data to increase case ascertainment to 91\%, effectively eliminating "response" bias. In addition, outcome data at 1, 2, 5 and 10 years were collated providing more robust measures of potential health effects than single time point outcomes could provide. Limitations of the study were that $49 \%$ and $23 \%$ of the cohort participated in the postal and clinical phases, compromising our ability to confidently comment on associations between maternal vitamin $\mathrm{D}$ and $\mathrm{E}$ and objective parameters of childhood asthma and atopy. Similarly, the absence of an association with the children's diet could be subject to Type II error because a relatively small number of children completed the children's FFQ. Participation was associated with significant biases; however, it is unlikely that these could account for the observed associations with maternal vitamin $\mathrm{D}$ and $\mathrm{E}$ intake because the nature of the biases was likely to weaken the observed associations rather than augment them. A further limitation was the lack of blood samples to measure 25 $(\mathrm{OH}) \mathrm{D}_{3}$ and our only vitamin $\mathrm{D}$ metric was an FFQ estimate that was reliant on subject recall of foods consumed and of their quantities. Although there was reasonable agreement between FFQ and diary-recorded intake for both vitamins D and E [27], FFQs can incur measurement error due to reporting bias and the use of standard portion size and food composition estimates for all respondents. However, such measurement error is usually random with respect to disease outcome and tends to attenuate associations, i.e. null bias. The focus of this study was maternal diet during pregnancy and accordingly when established we did not commence an expensive programme to capture numerous post-natal exposures that could influence asthma risk; however, these unquantified post-natal exposures are likely to attenuate any association between maternal diet and childhood outcomes, i.e. null bias. Although we adjusted for numerous potentially confounding factors, including those associated with the hygiene hypothesis and multiple metrics of SES, we cannot exclude the possibility of residual confounding by unmeasured or incorrectly measured covariates. Although we selected the primary outcome variables as those associated with maternal vitamin $\mathrm{D}$ and/or $\mathrm{E}$ intakes at 5 years, we still performed many tests of association, though these were not truly independent. Ultimately, issues of residual confounding, multiple testing and Type I errors will only be addressed by intervention studies. 
In the UK the mean vitamin $\mathrm{E}$ intake of women of childbearing age is about $8 \mathrm{mg} \cdot \mathrm{day}^{-1}$, with $93-97 \%$ having vitamin E intakes below the US National Institutes of Health recommended daily amount (RDA) of $15 \mathrm{mg} \cdot \mathrm{day}^{-1}$, and $80 \%$ below the European Union RDA of $10 \mathrm{mg} \cdot \mathrm{day}^{-1}$ [36]. There are no ongoing trials of vitamin E intervention during pregnancy with childhood wheeze, asthma and atopic outcomes. Notably however, antioxidant supplement trials for other conditions have failed to demonstrate beneficial effects of mono/oligo-nutrient supplements [37] and indeed a pre-eclampsia trial reported that unphysiologically high vitamin E supplementation $\left(133 \mathrm{mg} \cdot \mathrm{day}^{-1}\right)$ during pregnancy does not reduce the incidence of respiratory outcomes in 2-year-old children [38]. An alternative to supplements is a food-based intervention that increases vitamin $\mathrm{E}$ intake in the natural form of vitamin $\mathrm{E}$ containing foods, for example fat spreads, cereals, nuts, vegetables and meat. This embraces the complexity of diet by pragmatically accepting that the intervention not only increases vitamin $\mathrm{E}$ intake but also the intake of other naturally associated nutrients that may be contributing to the association. Indeed, a strength of such an intervention is that other nutrients (e.g. polyunsaturated fatty acids and vitamin D) or interactions between naturally associated nutrients may be essential for any beneficial effect to be preserved. We have piloted such a food-based intervention in pregnant women, successfully increasing maternal dietary vitamin $\mathrm{E}$ intake, but also increasing the intake of other nutrients reported to be beneficially associated with childhood respiratory outcomes in observational studies, namely polyunsaturated fatty acids, selenium and zinc [39].

The associations reported here support the hypothesis that children are more likely to wheeze and have asthma up to the age of 10 years if their mothers had low vitamin $\mathrm{D}$ and/or vitamin $\mathrm{E}$ intakes during pregnancy. However, it remains to be established if increasing vitamin D and E intakes is beneficial; meanwhile pregnant women should be advised to adhere to national policies on diet and supplements during pregnancy.

\section{References}

1 Devereux G. The increase in the prevalence of asthma and allergy: food for thought. Nat Rev Immunol 2006; 6: 869-874.

2 Camargo CA Jr, Rifas-Shiman SL, Litonjua AA, et al. Maternal intake of vitamin D during pregnancy and reports of recurrent wheezing in children at 3 y of age. Am J Clin Nutr 2007; 85: 788-795.

3 Erkkola M, Kaila M, Nwaru BI, et al. Maternal vitamin D intake during pregnancy is inversely associated with asthma and allergic rhinitis in 5-year-old children. Clin Exp Allergy 2009; 39: 875-882.

4 Nwaru BI, Ahonen S, Kaila M, et al. Maternal diet during pregnancy and allergic sensitization in the offspring by 5 yrs of age: a prospective cohort study. Pediatr Allergy Immunol 2010; 21: 29-37.

5 Miyake Y, Sasaki S, Tanaka K, et al. Dairy food, calcium, and vitamin D intake in pregnancy, and wheeze and eczema in infants. Eur Respir J 2010; 35: 1228-1234.

6 Maslova E, Hansen S, Jensen CB, et al. Vitamin D intake in mid-pregnancy and child allergic disease - a prospective study in 44,825 Danish mother-child pairs. BMC Pregnancy Childbirth 2013; 13: 199.

7 Wills AK, Shaheen SO, Granell R, et al. Maternal 25-hydroxyvitamin D and its association with childhood atopic outcomes and lung function. Clin Exp Allergy 2013; 43: 1180-1188.

8 Pike KC, Inskip HM, Robinson S, et al. Maternal late-pregnancy serum 25-hydroxyvitamin D in relation to childhood wheeze and atopic outcomes. Thorax 2012; 67: 950-956.

9 Morales E, Romieu I, Guerra S, et al. Maternal vitamin D status in pregnancy and risk of lower respiratory tract infections, wheezing, and asthma in offspring. Epidemiology 2012; 23: 64-71.

10 Magnus MC, Stene LC, Håberg SE, et al. Prospective study of maternal mid-pregnancy 25-hydroxyvitamin D level and early childhood respiratory disorders. Paediatr Perinat Epidemiol 2013; 27: 532-541.

11 Gale CR, Robinson SM, Harvey NC, et al. Maternal vitamin D status during pregnancy and childhood outcomes. Eur J Clin Nutr 2008; 62: 68-77.

12 Rothers J, Wright AL, Stern DA, et al. Cord blood 25-hydroxyvitamin D levels are associated with aeroallergen sensitization in children from Tucson, Arizona. J Allergy Clin Immunol 2011; 128: 1093-1099.

13 Weisse K, Winkler S, Hirche F, et al. Maternal and newborn vitamin D status and its impact on food allergy development in the German LINA cohort study. Allergy 2013; 68: 220-228.

14 Jones AP, Palmer D, Zhang G, et al. Cord blood 25-hydroxyvitamin D3 and allergic disease during infancy. Pediatrics 2012; 130: e1128-e1135.

15 Mullins RJ, Clark S, Wiley V, et al. Neonatal vitamin D status and childhood peanut allergy: a pilot study. Ann Allergy Asthma Immunol 2012; 109: 324-328.

16 Camargo CA Jr, Ingham T, Wickens K, et al. Cord-blood 25-hydroxyvitamin D levels and risk of respiratory infection, wheezing, and asthma. Pediatrics 2011; 127: e180-e187.

17 Baïz N, Dargent-Molina P, Wark JD, et al. Cord serum 25-hydroxyvitamin D and risk of early childhood transient wheezing and atopic dermatitis. J Allergy Clin Immunol 2014; 133: 147-153.

18 Litonjua AA, Rifas-Shiman SL, Ly NP, et al. Maternal antioxidant intake in pregnancy and wheezing illnesses in children at 2 y of age. Am J Clin Nutr 2006; 84: 903-911.

19 Miyake Y, Sasaki S, Tanaka K, et al. Consumption of vegetables, fruit, and antioxidants during pregnancy and wheeze and eczema in infants. Allergy 2010; 65: 758-765.

20 West CE, Dunstan J, McCarthy S, et al. Associations between maternal antioxidant intakes in pregnancy and infant allergic outcomes. Nutrients 2012; 4: 1747-1758.

21 Nwaru BI, Erkkola M, Ahonen S, et al. Intake of antioxidants during pregnancy and the risk of allergies and asthma in the offspring. Eur J Clin Nutr 2011; 65: 937-943. 
Maslova E, Hansen S, Strøm M, et al. Maternal intake of vitamins A, E and K in pregnancy and child allergic disease: a longitudinal study from the Danish National Birth Cohort. Br J Nutr 2014; 111: 1096-1108.

Devereux G, Litonjua AA, Turner SW, et al. Maternal vitamin D intake during pregnancy and early childhood wheezing. Am J Clin Nutr 2007; 85: 853-859.

24 Martindale S, McNeill G, Devereux G, et al. Antioxidant intake in pregnancy in relation to wheeze and eczema in the first two years of life. Am J Respir Crit Care Med 2005; 171: 121-128.

25 Devereux G, Turner SW, Craig LCA, et al. Reduced maternal vitamin E intake during pregnancy is associated with asthma in 5-year-old children. Am J Respir Crit Care Med 2006; 174: 499-507.

26 Scottish Collaborative Group. Food Frequency Questionnaire. www.foodfrequency.org Date last accessed: June, 2014.

27 Masson LF, McNeill G, Tomany JO, et al. Statistical approaches for assessing the relative validity of a food frequency questionnaire: use of correlation coefficients and the Kappa statistic. Public Health Nutr 2003; 6: 313-321.

28 Willett WC, Howe GR, Kushi LH. Adjustment for total energy intake in epidemiologic studies. Am J Clin Nutr 1997; 65: Suppl., 1220S-1228S.

29 The Scottish Government, National Statistics. Scottish Index of Multiple Deprivation. http://simd.scotland.gov.uk/ publication-2012 Date last accessed: May, 2014. Date last updated: December 18, 2012.

30 Nurmatov U, Nwaru BI, Devereux G, et al. Confounding and effect modification in studies of diet and childhood asthma and allergies. Allergy 2012; 67: 1041-1059.

31 Muehleisen B, Gallo RL. Vitamin D in allergic disease: shedding light on a complex problem. J Allergy Clin Immunol 2013; 131: 324-329.

32 Goldring ST, Griffiths CJ, Martineau AR, et al. Prenatal vitamin D supplementation and child respiratory health: a randomised controlled trial. PLoS One 2013; 8: e66627.

33 Cook-Mills JM, Abdala-Valencia H, Hartert T. Two faces of vitamin E in the lung. Am J Respir Crit Care Med 2013; 188: 279-284.

34 Joshi S, Kotecha S. Lung growth and development. Early Human Dev 2007; 83: 789-794.

35 Islam S, Narra V, Coté GM, et al. Prenatal vitamin E treatment improves lung growth in fetal rats with congenital diaphragmatic hernia. J Pediatr Surg 1999; 34: 172-176.

36 Henderson L, Irving K, Gregory J, et al. The National Diet \& Nutrition Survey: adults aged 19 to 64 years. Volume 3. Norwich, HMSO, 2003. http://tna.europarchive.org/20110116113217/http://www.food.gov.uk/ multimedia/pdfs/ndnsv3.pdf

37 Lawlor DA, Davey Smith G, Kundu D, et al. Those confounded vitamins: what can we learn from the differences between observational versus randomised trial evidence?. Lancet 2004; 363: 1724-1727.

38 Greenough A, Shaheen SO, Shennan A, et al. Respiratory outcomes in early childhood following antenatal vitamin $\mathrm{C}$ and E supplementation. Thorax 2010; 65: 998-1003.

39 Clark J, Craig L, McNeill G, et al. A novel dietary intervention to optimize vitamin E intake of pregnant women to $15 \mathrm{mg} /$ day. J Acad Nutr Diet 2012; 112: 297-301. 